\title{
Stochastic dynamics of the prisoner's dilemma with cooperation facilitators
}

\author{
Mauro Mobilia* \\ Department of Applied Mathematics, School of Mathematics, University of Leeds, Leeds LS2 9JT, United Kingdom
}

(Received 26 April 2012; published 30 July 2012)

\begin{abstract}
In the framework of the paradigmatic prisoner's dilemma game, we investigate the evolutionary dynamics of social dilemmas in the presence of "cooperation facilitators." In our model, cooperators and defectors interact as in the classical prisoner's dilemma, where selection favors defection. However, here the presence of a small number of cooperation facilitators enhances the fitness (reproductive potential) of cooperators, while it does not alter that of defectors. In a finite population of size $N$, the dynamics of the prisoner's dilemma with facilitators is characterized by the probability that cooperation takes over (fixation probability) by the mean times to reach the absorbing states. These quantities are computed exactly using Fokker-Planck equations. Our findings, corroborated by stochastic simulations, demonstrate that the influence of facilitators crucially depends on the difference between their density $z$ and the game's cost-to-benefit ratio $r$. When $z>r$, the fixation of cooperators is likely in a large population and, under weak selection pressure, invasion and replacement of defection by cooperation is favored by selection if $b(z-r)(1-z)>N^{-1}$, where $0<b \leqslant 1$ is the cooperation payoff benefit. When $z<r$, the fixation probability of cooperators is exponentially enhanced by the presence of facilitators but defection is the dominating strategy.
\end{abstract}

DOI: 10.1103/PhysRevE.86.011134

PACS number(s): 05.40.-a, 02.50.-r, 87.23.Kg, 87.23.Ge

\section{INTRODUCTION}

Understanding the origin of cooperative behavior is a central issue in the life and behavioral sciences and has recently been listed among the major scientific puzzles to be elucidated [1]. Evolutionary game theory (EGT) provides the ideal framework to study the competition between species and there is a long tradition of modeling the evolution of cooperation using evolutionary games [2,3]. In recent years, these processes have increasingly been investigated using the methods of statistical physics; see, for example, [2] and references therein. In EGT, successful species spread at the expense of the others, and each individual's reproductive potential (fitness) varies with the population's composition that continuously changes in time. The interaction between the species is thus accounted for by a fitness-dependent (or "frequency-dependent") selection pressure [2], as observed in various experiments [4]. Quite intriguingly, in such a setting the optimization of the fitness at an individual level can result in the reduction of the population overall fitness [2,3]. An influential example of such a paradoxical behavior is provided by the celebrated prisoner's dilemma (PD) game that serves as a metaphor for social dilemmas. In fact, in the classical PD individual interest leads to defection, even though mutual cooperation would be socially more beneficial $[2,3]$. While the PD is the paradigmatic model for the evolution of cooperation, its main prediction is at odds with the cooperative behavior that is commonly observed in experimental realizations [4,5]. This has motivated an upsurge of research aiming to identify the possible mechanisms capable of promoting cooperation in biological and social systems [6]. Notably it has been proposed that cooperation can be promoted by kin and group selection [7], as well as by conditional behavioral rules leading to direct or indirect reciprocity $[8,9]$. It has also been found

*M.Mobilia@leeds.ac.uk that local interactions may promote cooperation in some social dilemmas [10]. Furthermore, it has been shown that cooperation is supported in games with voluntary participation or with punishment for noncooperation [11].

In this work, we investigate an alternative scenario for the spread of cooperation in social dilemmas: we consider the evolution of the prisoner's dilemma in a finite population including a small number of "cooperation facilitators." The facilitators participate in the dynamics only by enhancing the reproductive potential of cooperators, while they do not affect the fitness of defectors (see Sec. II). To study the influence of cooperation facilitators on the prisoner's dilemma dynamics, the evolution is modeled in terms of a birth-death process and the fixation properties are studied analytically. In fact, it is well established that the evolutionary dynamics in finite populations is efficiently characterized by the probabilities of reaching the absorbing states, where the extinction of one or more species and the fixation of another occur [2,12-15]. Here, we are particularly interested in the probability that, from a given initial composition, the population eventually comprises only cooperators and a small fraction of facilitators, but no defectors ("cooperation fixation probability"). The mean times for these events (mean fixation times) are also studied, and our results are checked against stochastic simulations. This approach allows us to (i) discuss how demographic fluctuations alter the mean field predictions of the classic replicator equations [2] and (ii) thoroughly analyze the circumstances under which facilitators and selection favor single cooperators invading and replacing a population of defectors.

This paper is organized as follows: The PD with cooperation facilitators is introduced in the next section, where some of its properties are discussed. In Sec. III the dynamics with the Fermi process is characterized by the fixation probability (Sec. III A) and the mean fixation times (Sec. III B). The dynamics with the Moran process is studied in Sec. IV, while we summarize our findings and present our conclusions in Sec. V. 


\section{PRISONER'S DILEMMA WITH COOPERATION FACILITATORS: MODEL AND DYNAMICS}

In evolutionary game theory, two-player games can be interpreted as dilemmas of cooperation. In fact, the two possible strategies can be interpreted as "cooperation" (C) and "defection" (D). The paradigm of social dilemma is provided by the classical prisoner's dilemma, whose main features are captured by the following payoff matrix giving the pairwise interaction between cooperators and defectors $[2,3,10,16]$ :

$$
\begin{aligned}
& \text { C D } \\
& \mathrm{C}\left(\begin{array}{cc}
b-c & -c \\
b & 0
\end{array}\right) \text {, }
\end{aligned}
$$

where $b$ and $c$ respectively represent the benefit and the cost of cooperation, with $b>c>0$. Here, without loss of generality, we assume that $0<b \leqslant 1$. According to (1), mutual cooperation leads to payoff $b-c>0$ and mutual defection gives payoff 0 , whereas when one player defects and the other cooperates, the defector receives a payoff $b$ and the cooperators gets $-c$. In the (classical) PD, the dilemma arises from the fact that each individual is better off not cooperating, even though mutual cooperation enhances the population overall payoff. Hence, while cooperation is socially beneficial, defection is the only (strict) Nash equilibrium in the PD [2,3].

In this work, we consider a finite population comprising $N$ individuals on a complete graph (no spatial structure). The number of cooperators and defectors is respectively denoted by $j$ and $k$. In addition to cooperators and defectors, we consider that the population also comprises a fixed (small) number $\ell$ of "cooperation facilitators" $(\ell \ll N)$. These facilitators cooperate with $\mathrm{C}$ players and therefore enhance the reproductive potential (fitness) of cooperators, while they leave the fitness of defectors unaltered (see below). Hence, while the number of cooperators and defectors in the population changes in time ( $j$ and $k$ vary), the total number of cooperators and defectors $j+k=N-\ell$ is conserved. According to the tenets of EGT, the variation in time of the number of cooperators and defectors depends on their average payoffs, $\pi_{C}$ and $\pi_{D}$ respectively, obtained from the payoff matrix (1). Here, since facilitators enhance $\pi_{C}$ by cooperating with $C$ individuals and have no (direct) influence on $\pi_{D}$, one has

$$
\pi_{\mathrm{C}}=(b-c) \frac{j+\ell-1}{N-1}-c \frac{k}{N-1}, \quad \pi_{\mathrm{D}}=b \frac{j}{N-1},
$$

where we have excluded self-interactions from the definition of the payoffs [2]. The population average payoff is given by $\bar{\tau}=\left(j \pi_{\mathrm{C}}+k \pi_{\mathrm{D}}\right) / N$. It is worth noticing that the expression of $\pi_{\mathrm{C}}$ now comprises a term $(b-c) \ell /(N-1)>0$ reflecting the positive contribution of facilitators to the cooperators' payoff. In evolutionary dynamics, it is customary to add a baseline constant, here set to 1 , to the payoffs $\pi \mathrm{C} / \mathrm{D}$ of the spreading species $[2,13]$, yielding the fitness of species $C$ and $\mathrm{D}$, respectively given by

$$
\begin{aligned}
& f_{\mathrm{C}}=1+\pi_{\mathrm{C}}=1+b\left[\frac{j+\ell-r(N-1)-1}{N-1}\right], \\
& f_{\mathrm{D}}=1+\pi_{\mathrm{D}}=1+b \frac{j}{N-1},
\end{aligned}
$$

where we have introduced the cost-to-benefit ratio $r \equiv c / b$ (with $0<r<1$ ) and have used $k=N-j-\ell$. Similarly, the average fitness of the entire population reads $\bar{f}=\left(j f_{\mathrm{C}}+\right.$ $\left.k f_{\mathrm{D}}\right) / N=1+[b(1-r) j-\ell] / N$ and grows linearly with the density $x \equiv j / N$ of cooperators.

The size of the population being finite, the evolutionary dynamics is modeled as a continuous-time birth-death process $[2,17,18]$. In this model, only pairs of cooperators and defectors interact [according to Eq. (1)] and the stochastic dynamics is implemented as follows: (i) at each time step a pair of individuals is randomly chosen from the entire population; (ii) unless a pair of cooperator-defector is drawn, nothing happens; and (iii) if one picks a cooperator-defector pair, one of these individuals is randomly chosen for reproduction (proportionally to its fitness) and the other is replaced by newborn offspring. Hence, at each interaction the number of cooperators increases or decreases by one. The time evolution of this birth-death process can therefore be described by the random variable $j$ giving the number of cooperators and by the rates $T_{j}^{ \pm}$associated with the transitions $j \rightarrow j \pm 1$, respectively. Here, we consider

$$
T_{j}^{ \pm}=\frac{j(N-\ell-j)}{N(N-1)} \Psi^{ \pm}\left(f_{\mathrm{C}}, f_{\mathrm{D}}\right)
$$

where $j(N-\ell-j) / N(N-1)$ accounts for the probability of picking a cooperator-defector pair, while $\Psi^{ \pm}$are functions of the fitnesses (3) that encode the interactions (selection) according to the chosen "microscopic" update rule [2]. We here discuss the cases where $\Psi^{ \pm}$correspond to (i) the Fermi process (FP) $[19,20]$ and (ii) the Moran process (MP) $[2,12,14,20,21]$ that are commonly used in EGT [2].

Stochastic evolutionary dynamics and the influence of selection are generally characterized by the fixation properties, namely the probability that a given species fixates (takes over) the whole population by the mean time for such an event to occur $[2,12,13]$. In the absence of facilitators, fixation happens when only one species survives and the population composition is uniform. Here, as the number of facilitators remains constant, fixation will be achieved when one of the absorbing states is reached and either all cooperators are replaced by defectors, or vice versa, resulting in a (nonuniform) population comprising $\ell$ facilitators and $N-\ell$ cooperators or defectors. In this work, we are particularly interested in the probability $\phi_{j}^{C}$ that, starting with $j$ cooperators, all defectors are eventually removed from the population and replaced by cooperators. As discussed in Sec. III A, the fixation probability $\phi_{j}^{\mathrm{C}}$ is necessary to establish when selection favors cooperation replacing defection [13]. In the framework of the above birth-death process (4), this probability obeys the backward master equation $[2,14,18]$

$$
\phi_{j}^{\mathrm{C}}=T_{j}^{-} \phi_{j-1}^{\mathrm{C}}+T_{j}^{+} \phi_{j+1}^{\mathrm{C}}+\left[1-T_{j}^{-}-T_{j}^{+}\right] \phi_{j}^{\mathrm{C}},
$$

with absorbing boundaries $\phi_{0}^{\mathrm{C}}=0$ and $\phi_{N-\ell}^{\mathrm{C}}=1$. The formal solution of Eq. (5) reads [2,14,18]

$$
\phi_{j}^{\mathrm{C}}=\frac{1+\sum_{n=1}^{j-1} \prod_{m=1}^{n}\left(T_{j}^{-} / T_{j}^{+}\right)}{1+\sum_{n=1}^{N-\ell-1} \prod_{m=1}^{n}\left(T_{j}^{-} / T_{j}^{+}\right)} .
$$

Since the above birth-death process is a one-dimensional Markov chain, other quantities like the mean fixation times 
(MFTs) can, in principle, be obtained exactly, but yield unwieldy expressions $[14,18]$. When the population size $N$ is large, it is often much more useful to describe the fixation properties in terms of the diffusion approximation obtained in the continuum limit $(N \gg 1)$ by a second-order size expansion of the master equation, resulting in a Fokker-Planck equation $[12,15,17,18]$. By denoting $x \equiv j / N$ and $y \equiv k / N$ the initial density of cooperators and defectors, respectively; and with $z \equiv \ell / N$ being the fraction of facilitators in the population, the (backward) Fokker-Planck equation (FPE) associated with Eq. (5) reads $[17,18]$

$$
\mathcal{G}_{\text {back }}(x) \phi^{\mathrm{C}}(x)=0,
$$

where $\phi^{\mathrm{C}}(x) \equiv \phi_{j / N}^{\mathrm{C}}$ and

$$
\begin{aligned}
\mathcal{G}_{\text {back }}(x) \equiv & {\left[T^{+}(x)-T^{-}(x)\right] \frac{d}{d x} } \\
& +\frac{1}{2 N}\left[T^{+}(x)+T^{-}(x)\right] \frac{d^{2}}{d x^{2}},
\end{aligned}
$$

with $T^{ \pm}(x) \equiv T_{j / N}^{ \pm}$and, as usual, the density $x$ changes by $\pm \delta= \pm N^{-1}$ at each cooperator-defector interaction. In the realm of the Fokker-Planck equation, the time scale is such that the time step is $\delta=N^{-1}$. The formulation in terms of the FPE allows a neat connection with the mean field treatment of the dynamics. In fact, when $N \rightarrow \infty$ and all demographic fluctuations are negligible, the time variation of the density of cooperators is given by the drift term of Eq. (8) [17], that is,

$$
\begin{aligned}
\frac{d x(t)}{d t} & =T^{+}(x)-T^{-}(x) \\
& =x(1-z-x)\left[\Psi^{+}\left(f_{\mathrm{C}}, f_{\mathrm{D}}\right)-\Psi^{-}\left(f_{\mathrm{C}}, f_{\mathrm{D}}\right)\right] .
\end{aligned}
$$

As for the classic PD, this rate equation admits two absorbing fixed points, $x=0$ (no cooperators) and $x=$ $1-z$ (no defectors), but possesses no interior fixed point since $\Psi^{+}\left[f_{\mathrm{C}}(x), f_{\mathrm{D}}(x)\right] \neq \Psi^{-}\left[f_{\mathrm{C}}(x), f_{\mathrm{D}}(x)\right]$ for the Fermi and Moran processes. As discussed in what follows, the stability of these fixed points depends on the difference between the cost-to-benefit ratio $r$ and the fraction $z$ of facilitators.

\section{DYNAMICS WITH THE FERMI PROCESS}

The stochastic dynamics of evolutionary games is often conveniently modeled in terms of the so-called Fermi process (FP); see, for example, [19]. In the FP, at each time step two individuals are randomly drawn from the entire population and one of them reproduces at the expense of the other, that is replaced by the newborn offspring. This happens with a probability proportional to the difference between the fitness of the interacting individuals and given by the Fermi function from statistical physics. Since only the CD pairs interact, the dynamics with the FP is described by the birth-death process defined by Eq. (4) and $\Psi^{ \pm}=\left[1+e^{\mp\left(f_{\mathrm{C}}-f_{\mathrm{D}}\right)}\right]^{-1}=$ $\left[1+e^{\mp\left(\pi_{\mathrm{C}}-\pi_{\mathrm{D}}\right)}\right]^{-1}[19]$. With these expressions of $\Psi^{ \pm}$, one checks that $\Psi^{+}\left(f_{\mathrm{C}}, f_{\mathrm{D}}\right) \neq \Psi^{-}\left(f_{\mathrm{C}}, f_{\mathrm{D}}\right)$ [since $f_{\mathrm{C}} \neq f_{\mathrm{D}}$; see Eqs. (3) and (24)], which confirms the absence of an interior fixed point in the mean field (continuum) limit.

\section{A. Fixation probability}

With Eq. (3), the transition rates (4) for the Fermi process read

$$
T_{j}^{ \pm}=\frac{j(N-\ell-j)}{N(N-1)} \frac{1}{1+\exp \left( \pm v_{N}\right)},
$$

with

$$
v_{N} \equiv f_{\mathrm{D}}-f_{\mathrm{C}}=b\left[r-\left(z-\frac{1}{N}\right)\left(1+\frac{1}{N-1}\right)\right] .
$$

This quantity measures the selection pressure [22]. Clearly, $-b z<v_{N}<b(1-z)$ and $\left|v_{N}\right| \leqslant 1$. In the continuum limit $N \gg 1$, the densities $x=j / N, z=\ell / N$, and $v_{N} \rightarrow v \equiv$ $b(r-z)$ are treated as continuous quantities and the absence of self-interaction is ignored, yielding the transition rates (10)

$$
T^{ \pm}(x)=\frac{x(1-z-x)}{1+e^{ \pm v}} .
$$

The rate equation corresponding to the mean field dynamics with the Fermi process is obtained by using Eq. (12) in Eq. (9) and is characterized by a single stable (absorbing) fixed point corresponding to a stationary density

$$
x^{*}=\left\{\begin{array}{lll}
x_{\mathrm{C}}=1-z & \text { (no defectors) } & \text { if } v<0 \\
x_{\mathrm{D}}=0 & \text { (no cooperators) } & \text { if } v>0
\end{array}\right.
$$

of cooperators. This means that cooperation prevails $\left(x^{*}=x_{\mathrm{C}}\right.$, no defectors) in an infinitely large population comprising a fraction $z$ of facilitators higher than the cost-to-benefit ratio $r$, that is, when $v<0$. However, as in the traditional PD, defection wins ( $x^{*}=x_{\mathrm{D}}$, no cooperators) if $z$ is less than $r$ $(v>0)$. In other words, for cooperation to prevail $\left(x^{*}=x_{\mathrm{C}}\right)$ at mean field level, it is necessary that the density of facilitators compensates the cost of cooperation relative to its benefit.

When the population size is finite, demographic fluctuations are important and the evolution is thus no longer aptly described by the mean field dynamics (9). In particular, the mean field results (13) do not account for the nonzero probability that a single cooperator can invade and replace a (finite) population of defectors. Here, to investigate how the above mean field picture Eqs. (9) and (13) is altered by fluctuations arising in a finite population, we compute the probability $\phi_{j}^{C}$ that defection is eventually replaced by cooperation in a population comprising initially $j$ cooperators and $N-\ell-j$ defectors. Since $T_{j}^{+} / T_{j}^{-}=e^{v_{N}}$, this probability can be obtained explicitly using Eq. (6) and, when $v_{N} \neq 0$ [22], one finds

$$
\phi_{j}^{\mathrm{C}}=\frac{e^{j v_{N}}-1}{e^{N(1-z) v_{N}}-1},
$$

while fixation probability of defectors is simply given by $\phi_{j}^{\mathrm{D}} \equiv$ $1-\phi_{j}^{\mathrm{C}}=\left(e^{N(1-z) v_{N}}-e^{j v_{N}}\right) /\left(e^{N(1-z) v_{N}}-1\right)$. As shown in Fig. 1, where results of stochastic simulations obtained using the Gillespie algorithm [23] are reported, the predictions of Eq. (14) are in excellent agreement with numerical simulations. The expression (14) implies that

$$
\phi_{j}^{\mathrm{C}} \simeq \begin{cases}1-e^{-j\left|v_{N}\right|} & \text { if } \quad v_{N}<0 \\ \left(e^{j v_{N}}-1\right) e^{-N(1-z) v_{N}} & \text { if } \quad v_{N}>0,\end{cases}
$$




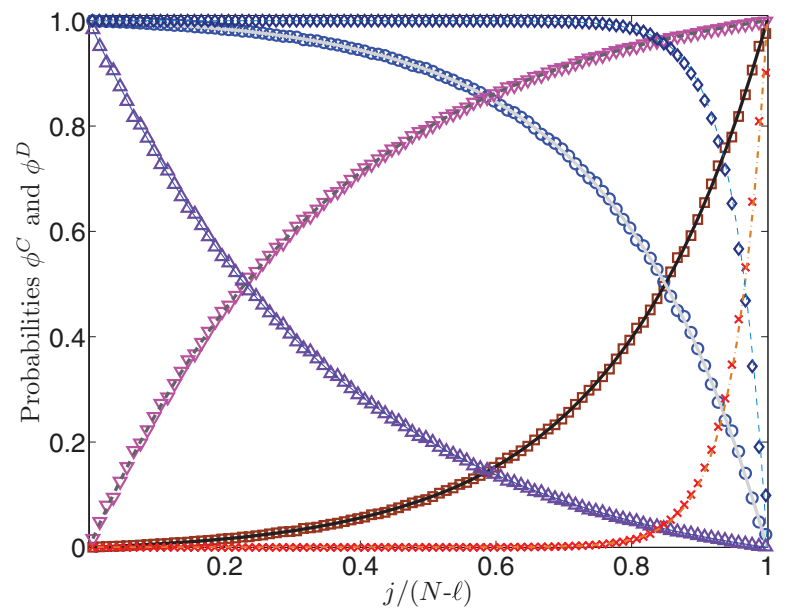

FIG. 1. (Color online) Probabilities $\phi_{j}^{\mathrm{C}}$ and $\phi_{j}^{\mathrm{D}}$ for various $z=\ell / N$ as function of $j /(N-\ell)=x /(1-z)$, and evolution with the Fermi process. Results of stochastic simulations (symbols) for $\phi_{j}^{\mathrm{C}}$ are compared with the predictions (curves) of Eq. (14) for $z=0$ ( $\times$, dot-dashed), 0.08 ( $\square$, solid), and 0.12 ( $\nabla$, dashed). Similarly for $\phi_{j}^{\mathrm{D}}$ with $z=0(\diamond$, thin dashed), 0.08 (०, solid gray), and 0.12 ( $\triangle$, thin solid). The other parameters are $N=200, b=1.0$, $c=0.1$ (i.e. $r=0.1$ ). Stochastic simulations for the birth-death process are defined by Eq. (10) and have been averaged over $2 \times 10^{5}$ samples.

when $N\left|v_{N}\right| \gg 1$. In particular, the cooperation fixation probability starting with a single cooperator reads

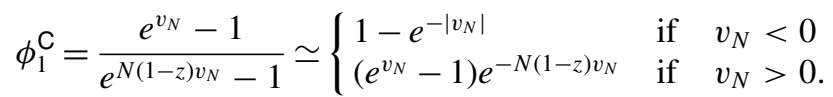

The findings (14)-(16), summarized in Fig. 1, illustrate how a small fraction $z$ of cooperation facilitators affects the fixation probabilities in a large, yet finite, population with an initial density of cooperators comparable to, or larger than, the density of defectors: When $v_{N}<0(z>r)$, the fixation probability of cooperators is much higher than that of defectors, $\phi_{j}^{\mathrm{C}} \gg \phi_{j}^{\mathrm{D}}$, and the spread of cooperation is thus efficiently promoted by facilitators. Yet, it is worth noticing that defectors still have finite probability to fixate even when $z>r$ (and $x \ll 1-z$ ), contrary to the mean field prediction (13). The opposite situation arises when $v_{N}>0(z<r)$, as shown in Fig. 1.

The results (15) and (16) can also be used to assess the influence of selection on the evolutionary dynamics [2]: Following the seminal work of Ref. [13], we can establish when selection favors cooperation $(\mathrm{C})$ invading and replacing defection (D). Selection is said to favor the replacement of $\mathrm{D}$ by $\mathrm{C}$ if the fixation probability $\phi_{1}^{\mathrm{C}}$ of a single cooperator in a population of $N-\ell-1$ defectors is greater than in the absence of selection pressure $\left(v_{N}=0\right)$ when $\phi_{1, v_{N}=0}^{\mathrm{C}}=(N-$ $\ell)^{-1}$ [22]. With Eq. (16), this yields the condition $1-e^{-\left|v_{N}\right|}>$ $[N(1-z)]^{-1}$ that is generally satisfied in large populations under nonvanishing selection pressure. An interesting result arises when the selection intensity is weak and the population size is large, that is, $\left|v_{N}\right| \rightarrow|v| \ll 1$ and $N \gg 1$. In such a limit, $\phi_{1}^{\mathrm{C}} \simeq|v|$ when $z>r$ (see Fig. 2 where $|v|=0.02$ ),

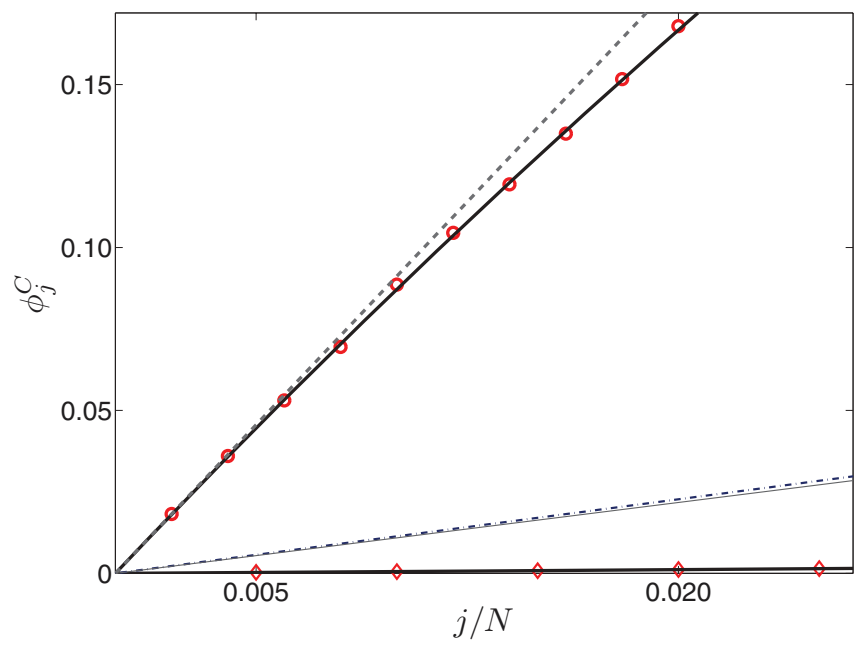

FIG. 2. (Color online) Probability $\phi_{j}^{\mathrm{C}}$ as function of $j / N$ when the initial number of cooperators is $j=1-10$ with $N=500$, and $j=1-5$ with $N=200$. The dynamics is implemented according to the Fermi process with Eq. (10). The results of stochastic simulations (symbols, averaged over $2 \times 10^{5}$ samples) are compared with Eq. (14) (curves/lines). Parameters are $b=1.0, c=$ 0.1 (i.e., $r=0.1)$, and $(N, z)=(500,0.12)(\circ),(200,0.08)(\diamond)$. Here, $\phi_{1}^{\mathrm{C}} \simeq 0.0182(\circ)$ and $\phi_{1}^{\mathrm{C}} \simeq 2.88 \times 10^{-4}(\diamond)$; see text. The dashed/dotted/thin lines correspond to $\phi_{j}^{C} \simeq j v_{N} /\left(e^{N(1-z) v_{N}}-1\right)$ with $(N, z)=(500,0.12)$ (dashed) and $\phi_{j, v_{N}=0}^{C}=j /(N-\ell)$ for $(N, \ell)=(200,16)$ (thin) and $(N, \ell)=(500,60)$ (dashed-dotted).

and selection favors cooperation replacing defection provided that $b(z-r)>[N(1-z)]^{-1}$. Moreover, selection favors $\mathrm{C}$ invading $\mathrm{D}$ when $f_{\mathrm{C}}>f_{\mathrm{D}}$ [13]. With Eq. (3), this yields the condition $z-r>(1-r) / N$. Therefore, under weak selection $(|v| \ll 1$ and $N \gg 1)$, selection favors the invasion and replacement of $\mathrm{D}$ by $\mathrm{C}$ if $z-r>\frac{1}{N} \max \left(1-r, \frac{1}{b(1-z)}\right)$. Since $0<b \leqslant 1$, one has $1-r \leqslant[b(1-z)]^{-1}$ and cooperation invading and replacing defection is favored by selection provided that

$$
b(z-r)(1-z)>N^{-1} .
$$

One can also use the results (16) to determine the circumstances under which defection is evolutionary stable. In fact according to [13], and as natural extension of the concept of evolutionary stability for infinitely large populations and deterministic evolutionary dynamics [2], D is evolutionary stable in a finite population if (i) selection opposes $\mathrm{C}$ invading $\mathrm{D}$, implying $f_{\mathrm{C}}<f_{\mathrm{D}}$ [i.e., $z-r<(1-r) / N$ ], and if (ii) selection opposes $\mathrm{C}$ replacing $\mathrm{D}$ [i.e., $\phi_{1}^{\mathrm{C}}<(N-\ell)^{-1}$ ]. The condition (ii) is clearly always satisfied when $\left|v_{N}\right|$ is finite, and in this case defection is evolutionary stable if $z-r<$ $(1-r) / N$. In the weak selection limit where $|v| \ll 1$ (with $N \gg 1$ ), the condition (ii) yields $z-r<[b N(1-z)]^{-1}$. Hence, defection is evolutionary stable under weak selection in a large population if $z-r<\frac{1}{N} \min \left(1-r, \frac{1}{b(1-z)}\right)=$ $(1-r) / N$. Since $r<1$, this clearly means that defection is evolutionary stable and is the dominating strategy when $z<r$. It is worth noticing that in the limit of an infinite population, $N \rightarrow \infty$, one recovers the mean field results (13): Cooperation prevails only if $z>r$, according to Eq. (17), and defection dominates otherwise. 
The meaning of the results (15)-(17) is illustrated in Fig. 2 where $\phi_{j}^{\mathrm{C}}$ has been computed in populations comprising a small initial number of cooperators $(j=1, \ldots, 10)$ and excellent agreement with Eqs. (15) and (16) has been found. In Fig. 2, $\left|v_{N}\right| \ll 1$ and we notice that $\phi_{j}^{\mathrm{C}}$ increases linearly in $x=j / N \ll 1$, with a slope steeper than $(1-z)^{-1}$ when $z>r$ and selection favors cooperation replacing defection. The slope is less than $(1-z)^{-1}$ when $z<r$ and the fixation of cooperation is opposed by selection. To further appreciate the implications of Eqs. (14)-(17), it is useful to compare these results with those obtained in the absence of facilitators. Putting $z=0$ in Eqs. (14)-(17), one recovers the results for the classic PD when cooperation fixation probability vanishes exponentially with the population size $N: \phi_{j, z=0}^{\mathrm{C}} \sim e^{-(N-j) c}$ and $\phi_{1, z=0}^{\mathrm{C}} \sim e^{-N c}[2,14]$ (see Fig. 1).

Our findings therefore demonstrate that facilitators greatly influence the probability that cooperation prevails and are summarized in Fig. 1. As illustrated in that figure, the influence of facilitators crucially depends on the difference between their density $z$ and the cost-to-benefit ratio $r$ :

(i) When $v_{N}<0$, the fixation of cooperators is likely (but not certain) even when they are initially in minority, that is, even when initially $x=j / N<1 / 2$.

(ii) When $v_{N}<0$ and $N\left|v_{N}\right| \gg 1$, the fixation probability of a single cooperator is generally higher than in the absence of selection pressure $\left(v_{N}=0\right)$. In this case, with $z>r$, selection favors cooperation invading and replacing defection; see Eq. (16) and Figs. 1 and 2. Furthermore, under weak selection pressure and in a large population $(|v| \ll 1$ and $N \gg 1)$, the fixation probability of a single cooperator is independent of $N$, $\phi_{1}^{\mathrm{C}} \simeq z-r$. In this case invasion and replacement of defection by cooperation is favored by selection if Eq. (17) is satisfied.

(iii) When $v_{N}>0$, selection always opposes cooperation replacing defection. In this case, while defection is evolutionary stable and is the dominating strategy when $z<r$, the cooperation fixation probability is exponentially enhanced by a small fraction of facilitators. Yet, cooperation is likely to fixate only if defectors are initially outnumbered by cooperators, that is, if $j \gg k$, as illustrated in Fig. 1.

\section{B. Mean fixation times}

Another quantity of great interest to unveil the influence of facilitators in the evolutionary dynamics of the PD is the (unconditional) mean fixation time. This quantity gives the average time necessary to reach one of the absorbing boundaries, that is, a population composition with either 0 or $N-\ell$ cooperators. The unconditional mean fixation time (MFT), $\tau_{j}$, for a system comprising initially $j$ cooperators obeys the following backward master equation $[2,14,18]$ (where the time step is $\delta=N^{-1}$ ):

$$
\tau_{j}=\delta+T_{j}^{-} \tau_{j-1}+T_{j}^{+} \tau_{j+1}+\left[1-T_{j}^{-}-T_{j}^{+}\right] \tau_{j},
$$

with boundary conditions $\tau_{0}=\tau_{N-\ell}=0$. In principle, this equation can be solved exactly but the final result is cumbersome and not enlightening. Here, in the continuum limit $N \gg 1$, we work with the continuous quantities $x=j / N$, $z=\ell / N$ and $v=b(r-z)$, and adopt the approach of diffusion theory [12,17]. The diffusion approximation is known to be particularly suited to analyze the dynamics under weak selection, which here corresponds to the regime where $|v| \ll 1[2,13,15]$. Exact methods (when available) or other approximations [14], for example, the WKB approach [20], are particularly useful to deal with the case of strong selection intensity and/or with phenomena like metastability. In the realm of the diffusion theory, the transition rates of the FP are given by Eq. (12) and the fixation probability of cooperation is obtained by solving Eq. (8), which yields

$$
\phi^{\mathrm{C}}(x)=\frac{e^{N v x}-1}{e^{N(1-z) v}-1},
$$

while for defection the probability is $\phi^{\mathrm{D}}(x)=1-\phi^{\mathrm{C}}(x)$.

Similarly, the unconditional MFT is obtained by solving the backward FPE $\mathcal{G}_{\text {back }}(x) \tau(x)=-1[15,17]$, that is,

$$
x(1-z-x)\left[\tanh \left(\frac{v}{2}\right) \frac{d}{d x}-\frac{1}{2 N} \frac{d^{2}}{d x^{2}}\right] \tau(x)=1,
$$

with the absorbing boundary conditions $\tau(0)=\tau(1-z)=0$. When the drift and diffusive terms are of the same order (i.e., when $|v| \sim N^{-1} \ll 1$ ), it follows from Eq. (19) that the MFT scales linearly with $N$ :

$$
\tau(x)=N \mathcal{F}_{v}(x) .
$$

The scaling function can be obtained explicitly by solving Eq. (19) using standard methods; see, for example, [17]. For instance, when the initial density of cooperators and defectors is the same, $x=y=(1-z) / 2$ and $|v| \sim N^{-1} \ll 1$, one finds

$$
\begin{aligned}
\mathcal{F}_{v}\left(\frac{1-z}{2}\right)= & \frac{e^{-(2+z) q}}{q(1-z)\left(1+e^{-q(1-z)}\right)} \\
& \times\left[e^{(2+z) q}\left\{\gamma_{\mathrm{E}}-\ln 2-\operatorname{Ei}[-(1-z) q]\right\}\right. \\
& +e^{(1+2 z) q}\left\{\operatorname{Ei}[(1-z) q]-\gamma_{\mathrm{E}}-\ln (2 q)\right\} \\
& +e^{3 z q}\{\operatorname{Ei}[(1-z) q]-\operatorname{Ei}[2(1-z) q]\} \\
& +e^{3 q}\{\operatorname{Ei}[-2(1-z) q]-\operatorname{Ei}[-(1-z) q]\} \\
& \left.+e^{(1+z) q}\left(e^{q}-e^{z q}\right) \ln (1-z)\right],
\end{aligned}
$$

where $q \equiv N|\tanh (v / 2)| \simeq N|v| / 2, \operatorname{Ei}(x) \equiv \int_{-\infty}^{x} \frac{e^{u}}{u} d u$ denotes the usual exponential integral, and $\gamma_{\mathrm{E}}=0.5772 \ldots$ is the Euler-Mascheroni constant. While the expression of $\mathcal{F}_{v}$ is usually cumbersome, some useful properties can be directly inferred from Eq. (19). In fact, as Eq. (19) is invariant under the transformation $(x, r) \rightarrow(1-z-x, 2 z-r)$, one has $\mathcal{F}_{v}(x)=$ $\mathcal{F}_{-v}(1-z-x)$ when $z$ is kept fixed. The unconditional MFT in the Fermi process is therefore characterized by the symmetry

$$
\left.\tau(x)\right|_{r}=\left.\tau(1-z-x)\right|_{r \rightarrow r^{\prime}=2 z-r},
$$

where on the right-hand side $r$ is replaced by $r^{\prime}=2 z-r$ and $v$ is transformed into $-v$, with $z$ kept fixed. Furthermore, when $r=c / b$ is kept fixed but $z$ varies, Eq. (19) is invariant under the transformation $z \rightarrow z^{\prime}=2 r-z$ and $x \rightarrow 1-z^{\prime}-x$, while the boundary conditions become $\tau(1-$ $\left.z^{\prime}\right)=0$ and $\tau\left(z-z^{\prime}\right)=\tau(-2 v / b)=0$. In the weak selection regime $|v| / b=|z-r| \ll 1$, the second boundary condition can be approximated by $\tau\left(z-z^{\prime}\right) \simeq \tau(0)=0$, which allows a mapping onto (19) that yields

$$
\left.\left.\tau(x)\right|_{z} \simeq \tau\left(1-z^{\prime}-x\right)\right|_{z \rightarrow z^{\prime}=2 r-z},
$$

with $r=c / b$ fixed. The comparison between the solution of Eq. (19) and the results of stochastic simulations [for the FP 


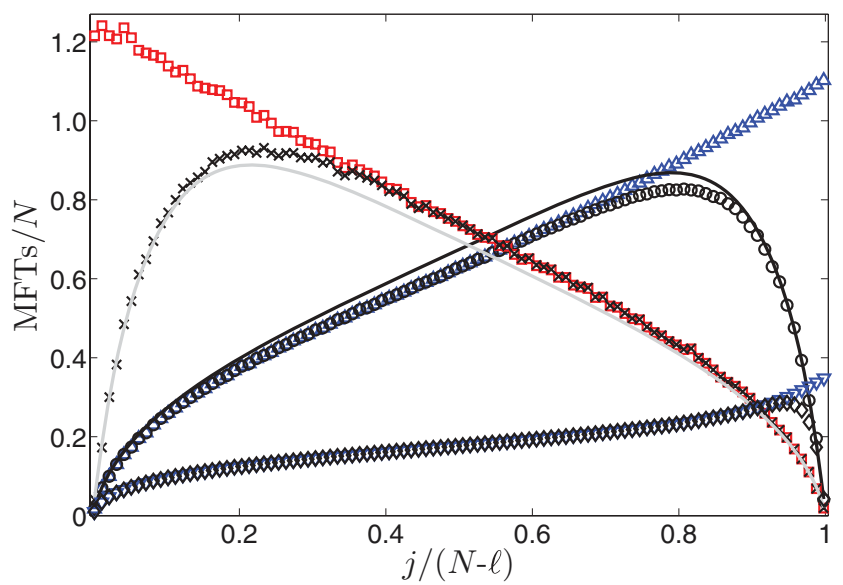

FIG. 3. (Color online) Mean fixation times as function of $j$ / $(N-\ell)=x /(1-z)$ for the evolution with the Fermi process. Results of stochastic simulations (symbols) for $\tau$ are compared with the solution (curves) of Eq. (19) for $z=0(\diamond), 0.08$ ( $\circ$, solid black), and $0.12(\times$, solid gray). We also report the numerical results for the conditional MFTs $\tau^{\mathrm{C}}$ for $z=0.12(\square)$ and $\tau^{\mathrm{D}}$ with $z=\ell / N=$ $0(\nabla), 0.08(\triangle)$. The other parameters are $N=500, b=1.0, c=0.1$ (i.e. $r=0.1$ ). Stochastic simulations are for the FP with rates (10) and have been averaged over $2 \times 10^{5}$ samples.

with rates (10)] reported in Fig. 3 shows that the diffusion approximation aptly captures the functional dependence of $\tau$, even though some deviations (of about 10\%) can be noticed. These deviations stem from the self-interaction terms that are excluded from Eq. (10) but not in the continuum limit (12) [e.g., in Fig. 3 one has $v_{N} \simeq-0.0182$ and $v=-0.02$ when $z=0.12$, and $v_{N} \simeq 0.0218$ and $v=0.02$ for $\left.z=0.08\right]$. More importantly, the scaling (20) and the relationship (23) are confirmed by the numerical simulations of Fig. 3. In fact, in Fig. 3 we notice that $\tau(x)$ is a humped function with a maximum well separated from the absorbing boundaries and located at $x /(1-z)<1 / 2$ when $z>r$ and, while $\tau$ scales linearly with $N$, the presence of facilitators increases the unconditional MFT and its maximum value at the hump.

In addition to the unconditional MFT, it is also relevant to consider the mean time to specifically reach one of the absorbing boundaries. Hence, the conditional mean fixation times $\tau^{\mathrm{C}}(x)$ and $\tau^{\mathrm{D}}(x)$ respectively give the average time to reach the absorbing boundaries $x=1-z$ and $x=0$ [14,20]. As for the unconditional MFT, these quantities can be obtained from a backward FPE in the realm of the diffusion approximation. In fact, $\tau^{\mathrm{C}}(x)$ obeys $\mathcal{G}_{\text {back }}(x)\left[\phi^{\mathrm{C}}(x) \tau^{\mathrm{C}}(x)\right]=-\phi^{\mathrm{C}}(x)$, with the absorbing boundaries $\phi^{\mathrm{C}}(1-z) \tau^{\mathrm{C}}(1-z)=\phi^{\mathrm{C}}(0) \tau^{\mathrm{C}}(0)=0$ [12]. Since $\phi^{\mathrm{D}}(x)=1-\phi^{\mathrm{C}}(x)$ and, from Eq. (14), $\phi^{\mathrm{D}}(x)=$ $\phi^{\mathrm{C}}[1-(2 r-z)-x]$, the conditional MFTs in the regime $|v| / b \ll 1$ (weak selection) are related by the relationship $\left.\left.\tau^{\mathrm{C}}(1+z-2 r-x)\right|_{z} \simeq \tau^{\mathrm{D}}(x)\right|_{z \rightarrow z^{\prime}=2 r-z}$ where $r$ is kept fixed, as illustrated in Fig. 3. Furthermore, one has $\phi^{\mathrm{D}}(x) \simeq 1$ when $x \rightarrow 0$ and $v>0(z<r)$, while $\phi^{\mathrm{C}}(x) \simeq 1$ when $x \rightarrow 1$ and $v<0(z>r)$. This implies that

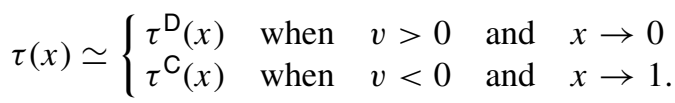

As shown in Fig. $3, \tau^{\mathrm{C}}(x)$ decreases while $\tau^{\mathrm{D}}(x)$ increases monotonically with $x /(1-z)$.

The influence of facilitators on the unconditional and conditional MFTs is summarized in Fig. 3. We have found that in the PD with cooperation facilitators, all conditional and unconditional MFTs scale linearly with the population size $N$ when $|v| \sim N^{-1}$ (weak selection). While a similar scaling is also obtained in the absence of facilitators, the MFTs at a fixed value $x /(1-z)$ are found to be significantly increased by the presence of facilitators. Hence, the presence of cooperation facilitators has the quantitative effect to prolong the coexistence and the competition between cooperators and defectors before an absorbing state is reached; see Fig. 3.

\section{DYNAMICS WITH THE FITNESS-DEPENDENT MORAN PROCESS}

The stochastic dynamics of evolutionary games is often implemented in terms of the Moran process (see, e.g., $[2,13]$ ) that was originally introduced in population genetics $[12,21]$. In its essence, the Moran model is a birth-death process where one randomly picked individual produces an offspring proportionally to its fitness relative to the population average fitness. The resulting offspring then replaces another individual that is randomly picked to be removed from the population, whose size is therefore conserved. Here, as the interactions are between cooperators and defectors, the Moran process is implemented with $\Psi^{+}=f_{\mathrm{C}} / \bar{f}$ and $\Psi^{-}=f_{\mathrm{D}} / \bar{f}$ in Eq. (4). Since $f_{\mathrm{C}} \neq f_{\mathrm{D}}$ when $v \neq 0$ [see Eq. (24) and [22]], one verifies that $\Psi^{+}\left(f_{\mathrm{C}}, f_{\mathrm{D}}\right) \neq \Psi^{-}\left(f_{\mathrm{C}}, f_{\mathrm{D}}\right)$, implying the absence of an interior fixed point in the mean field (continuum) limit. The Moran process is usually investigated when the selection intensity is weak, both for technical convenience (the mathematical treatment simplifies greatly) and for the biological relevance of such a limit $[2,12,13]$. In this section, the stochastic dynamics with the Moran process is investigated in the weak selection limit, where $|v|=b|r-z| \ll 1$, using the diffusion approximation.

\section{A. Fixation probability}

In the continuum limit, the fitnesses (3) become

$$
f_{\mathrm{C}}(x)=1-v+b x \quad \text { and } \quad f_{\mathrm{D}}(x)=1+b x,
$$

with $\bar{f}(x)=1-z+b(1-r) x$. The transition rates for the Moran process thus read

$$
T^{+/-}(x)=x(1-z-x) \frac{f_{\mathrm{C} / \mathrm{D}}(x)}{\bar{f}(x)} .
$$

With Eqs. (24) and (25), the mean field dynamics is described by the rate equation (9), whose properties are similar to those discussed for the Fermi process. In particular, the rate equation (9) for the Moran process is also characterized by a single stable (absorbing) fixed point $x^{*}=x_{\mathrm{C}}$ (no defectors) if $v<0$ and $x^{*}=x_{\mathrm{D}}$ (no cooperators) if $v>0$ [see (13)].

To understand how the combined effects of nonlinear selection and demographic noise alter the mean field description, we now compute the cooperation fixation probability in the realm of the diffusion approximation. In such a setting, the fixation 
probability $\phi^{\mathrm{C}}(x)$ is given by the FPE [Eqs. (7) and (8)] with the boundary conditions $\phi^{\mathrm{C}}(0)=0$ and $\phi^{\mathrm{C}}(1-z)=1$. The solution of Eq. (7) is given by [17]

$$
\phi^{\mathrm{C}}(x)=\frac{\int_{0}^{x} d u \chi(u)}{\int_{0}^{1-z} d u \chi(u)},
$$

where, with Eqs. (24) and (25),

$$
\begin{aligned}
\chi(u) & =\exp \left(-2 N \int_{0}^{u} d s\left\{\frac{T^{+}(s)-T^{-}(s)}{T^{+}(s)+T^{-}(s)}\right\}\right) \\
& =\exp \left(2 N v \int_{0}^{u} \frac{d s}{2 b s+2-v}\right) .
\end{aligned}
$$

By introducing Eq. (27) into Eq. (26) and performing the integrals, one obtains

$$
\phi^{\mathrm{C}}(x)=\frac{\left(1+\frac{2 b}{2-v} x\right)^{1+N v / b}-1}{\left[1+\frac{2 b}{2-v}(1-z)\right]^{1+N v / b}-1} .
$$

As shown in Fig. 4, this result is in excellent agreement with numerical simulations and exhibits the same qualitative features obtained for the Fermi process (compare with Fig. 1). The finding (28) implies that in the weak selection limit where $|v| \ll 1$ and $N|v| \gg 1$, one has

$$
\phi^{\mathrm{C}}(x) \simeq\left\{\begin{array}{lll}
1-(1+b x)^{-N(z-r)} & \text { if } & z>r \\
\left(\frac{1+b\left(x-\frac{z-r}{2}\right)}{1+b\left(1-\frac{z+r}{2}\right)}\right)^{N(r-z)} & \text { if } & r>z .
\end{array}\right.
$$

In particular, the probability that cooperation fixates starting with a single cooperator, when $z>r$ is given by $\lim _{N x \rightarrow 1} \phi^{\mathrm{C}}(x)=1-e^{-|v|} \simeq|v|$. We therefore recover the result derived from Eq. (16) for the Fermi process. Clearly, this implies that under weak selection the fixation of a single cooperator is favored by selection if the nontrivial condition (17) is satisfied. Again, it is instructive to compare Eqs. (28) and (29) with the result obtained in the absence of facilitators, when $\left.\phi^{\mathrm{C}}(x)\right|_{z=0} \simeq\left(\frac{1+b(x-r / 2)}{1+b(1-r / 2)}\right)^{N r}$ decays to zero exponentially with $N$. The influence of the facilitators on the fixation probabilities for the Moran process is summarized in Fig. 4, where the same features as in Fig. 1 are recognized and summarized as follows:

(i) The fixation of cooperators is likely (but not certain) when the density of facilitators is higher than the cost-tobenefit ratio $(z>r)$.

(ii) When $|v| \ll 1$ and $N|v| \gg 1$, selection favors cooperation invading and replacing defection if Eq. (17) is satisfied. In particular, the fixation probability of a single cooperator is $\lim _{N x \rightarrow 1} \phi^{\mathrm{C}}(x) \simeq|v|$.

(iii) When $z<r$, selection opposes cooperation replacing defection but the fixation probability of cooperators is exponentially enhanced by the presence of facilitators.

\section{B. Mean fixation times}

In the realm of the diffusion approximation, the unconditional mean fixation time $\tau$ obeys the backward FPE $\mathcal{G}_{\text {back }}(x) \tau(x)=-1$, with the absorbing boundary conditions $\tau(0)=\tau(1-z)=0$. In the weak selection regime $c<b \ll 1$

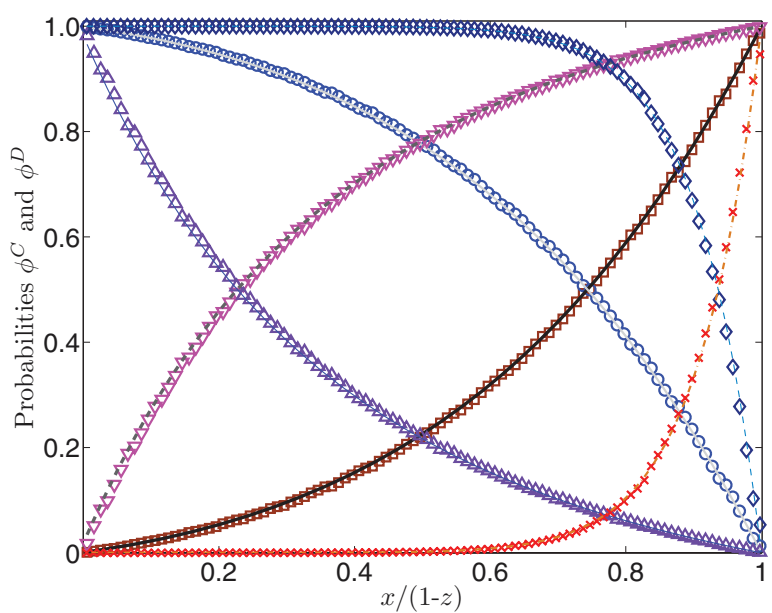

FIG. 4. (Color online) Probabilities $\phi^{\mathrm{C}}(x)$ and $\phi^{\mathrm{D}}(x)$ for various $z$ as functions of $x /(1-z)$, and the dynamics with the Moran process (25). Results of stochastic simulations (averaged over $2 \times 10^{5}$ samples) are compared with Eq. (28) for $z=0(\times$, dot-dashed), 0.08 ( $\square$, solid), and $0.12\left(\nabla\right.$, dashed). Similarly for $\phi^{\mathrm{D}}(x)$ with $z=0$ ( $\diamond$, thin dashed), 0.08 ( $\circ$, solid gray), and $0.12(\triangle$, thin solid). The other parameters are $N=200, b=1.0$, and $c=0.1(|v|=0.02)$.

and continuum limit, with Eq. (25), one has

$$
\begin{aligned}
& T^{+}(x)-T^{-}(x) \simeq-\frac{v}{1-z} x(1-z-x), \\
& T^{+}(x)+T^{-}(x) \simeq \frac{2}{1-z} x(1-z-x) .
\end{aligned}
$$

With these expression, the backward FPE for the unconditional MFT reads

$$
\frac{x(1-z-x)}{1-z}\left[-v \frac{d}{d x}+\frac{1}{N} \frac{d^{2}}{d x^{2}}\right] \tau(x)=-1,
$$

with $\tau(0)=\tau(1-z)=0$. When $|v|, b \ll 1$, Eq. (30) coincides with the FPE (19) for the Fermi process with an effective population size $N(1-z) / 2$. The solution to Eq. (30) can therefore readily be obtained from Eqs. (19) and (20). In particular, we infer from Eq. (20) that the MFT scales linearly with $N(1-z) / 2$ when $|v| \sim N^{-1}$, yielding

$$
\tau(x)=\frac{N(1-z)}{2} \mathcal{F}_{v}(x),
$$

where $\mathcal{F}_{v}(x)$ is the scaling function (20) obtained for the Fermi process. This function still satisfies the symmetry $\mathcal{F}_{v}(x)=\mathcal{F}_{-v}(1-z-x)$, yielding $\left.\tau(x)\right|_{r}=\tau(1-z-$ $x)\left.\right|_{r \rightarrow r^{\prime}=2 z-r}$ when $z$ is kept fixed, as in the Fermi process.

In the same manner, from Eqs. (31) and (23), we infer

$$
\left.\tau(x) \simeq\left(\frac{1-z}{1+z-2 r}\right) \tau\left(1-z^{\prime}-x\right)\right|_{z \rightarrow z^{\prime}=2 r-z}
$$

when $r=c / b$ is kept fixed and $z$ is transformed into $z^{\prime}=$ $2 r-z$. The solution of Eq. (30), as well as the relationships Eqs. (31) and (32), are in excellent agreement with the results of stochastic simulations reported in Fig. 5. As for the FP, we 


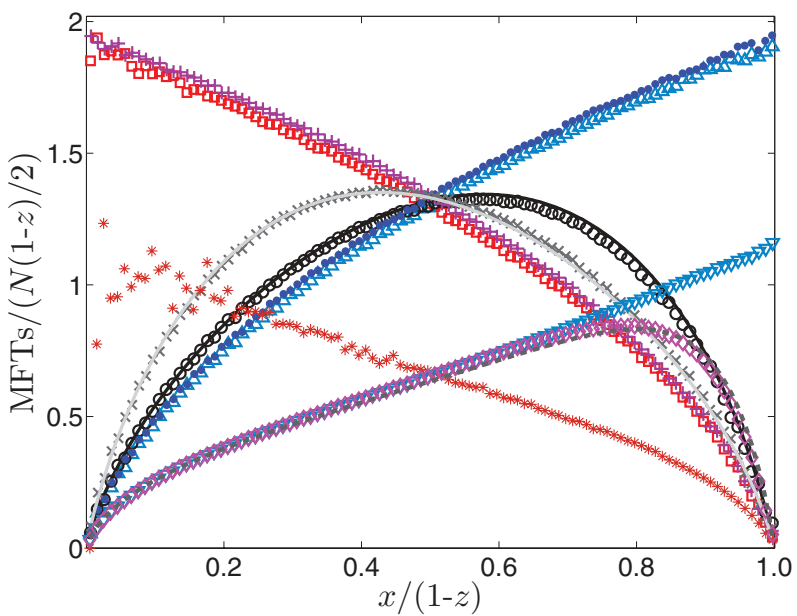

FIG. 5. (Color online) Rescaled mean fixation times as function of $x /(1-z)$ for the evolution with the Moran process (12). Results of stochastic simulations for $\tau$ are compared with (30) for $z=0(\diamond$, dashed), 0.16 ( $\circ$, solid black curve), and $0.24(\times$, solid gray). Numerical results for the conditional MFTs $\tau^{\mathrm{C}}$ with $z=0(*), 0.16(\square)$, and $0.24(+)$ and for $\tau^{\mathrm{D}}$ with $z=0(\nabla)$, $0.16(\triangle)$, and $0.24(\bullet)$. The parameters are $N=200, b=0.25$, and $c=0.05(r=0.2$ and $|v|=0.04)$. Stochastic simulations have been averaged over $2 \times 10^{5}$ samples.

can also consider the conditional mean fixation times and it follows from Eqs. (31) and (23) that for the Moran process the conditional MFTs are related by $\left(\frac{1+z-2 r}{1-z}\right) \tau^{\mathrm{C}}(1+z-2 r-$ $x)\left.\left.\right|_{z} \simeq \tau^{\mathrm{D}}(x)\right|_{z \rightarrow z^{\prime}=2 r-z}$ where $r$ is kept fixed, in agreement with the results of Fig. 5.

The influence of facilitators on the MFTs with the Moran process is summarized in Fig. 5, where the MFTs rescaled by a factor $[N(1-z) / 2]^{-1}$ reproduce the same qualitative behavior obtained for the Fermi process (compare with Fig. 3) and $\tau(x)$ is a humped function with a pronounced maximum. Again, all MFTs scale linearly with $N$ (in the weak selection limit). Yet, the comparison with the results for $z=0$ reveals that at a fixed value of $x /(1-z)$, the presence of facilitators increases the MFTs; see Fig. 5. Also, we notice that the monotonic dependence of $\tau^{\mathrm{C}}$ and $\tau^{\mathrm{D}}$ on $x$ is essentially independent of the sign of $v \neq 0$ (in Fig. 5, $v= \pm 0.04$ and $v=0$ ).

\section{SUMMARY AND CONCLUSION}

In this work, we have proposed and investigated an alternative scenario leading to the spread of cooperation in social dilemmas. We have considered the evolutionary dynamics of the prisoner's dilemma (PD) game in the presence of a small number of cooperation facilitators. These individuals participate in the dynamics only by enhancing the fitness of cooperators. The influence of facilitators on the evolutionary dynamics has been characterized by computing the model's fixation properties in a finite population of size $N$. Here, fixation occurs either in the state with only defectors (as in the classic PD) or in the state where the entire population is composed of cooperators and facilitators. The dynamics has been implemented with the Fermi and Moran processes and the same qualitative results have been found, which demonstrates the robustness of our findings. Our analytical approach, corroborated by stochastic simulations, is based on an exact treatment and on the diffusion approximation (Fokker-Planck equation) of the underlying birth-death process.

Our main results concern the fixation probabilities, whose properties crucially depend on whether the fraction of facilitators $z$ is more or less than the game's cost-to-benefit ratio $r$. When $z>r$, we have shown that facilitators are very efficient in promoting the spread of cooperators whose fixation is likely (but not certain, contrary to the mean field predictions) in a large population with comparable initial densities of defectors and cooperators. Furthermore, when the selection intensity is weak and $N \gg 1$, we have demonstrated that the invasion and replacement of defectors by a single cooperator is favored by facilitators and selection if $b(z-r)(1-z)>N^{-1}$ (where $0<b \leqslant 1$ is the cooperation payoff benefit). When $z<r$, defection is evolutionary stable and the dominating strategy. In this case, while cooperation is unlikely to fixate, the cooperators fixation probability is still exponentially enhanced by the presence of facilitators. We have also studied the (unconditional and conditional) mean fixation times in the weak selection limit and found that these quantities grow linearly with the population size. While a similar scaling is also obtained in the absence of facilitators, their presence has the effect of significantly increasing all the mean fixation times and hence prolonging the coexistence of cooperators and defectors.

In conclusion, this work demonstrates that the presence of a small number of cooperation facilitators can effectively enhance the spread of cooperation in a simple model of social dilemmas and prolong the coexistence of competing species. The influence of facilitators is particularly drastic when their abundance exceeds the game's cost-to-benefit ratio, in which case cooperation is generally the strategy favored by selection in large populations. These findings pave the way to further investigations of the influence of facilitators in other social dilemmas, for example, with mixed strategies and/or in spatial settings.
[1] E. Pennisi, Science 309, 90 (2005).

[2] J. Maynard Smith, Evolution and the Theory of Games (Cambridge University Press, Cambridge, 1982); J. Hofbauer and K. Sigmund, Evolutionary Games and Population Dynamics (Cambridge University Press, Cambridge, 1998); H. Gintis, Game Theory Evolving (Princeton University Press, Princeton, NJ, 2000); M. A. Nowak, Evolutionary Dynamics (Belknap
Press, Cambridge, MA, 2006); G. Szabó and G. Fáth, Phys. Rep. 446, 97 (2007); A. Traulsen and C. Hauert, in Reviews of Nonlinear Dynamics and Complexity, edited by H.-G. Shuster, Vol. 2 (Wiley-VCH, New York, 2010).

[3] R. Axelrod and W. D. Hamilton, Science 211, 1390 (1981); R. Axelrod, The Evolution of Cooperation (Basic Books, New York, 1984). 
[4] P. E. Turner and L. Chao, Nature (London) 398, 441 (1999); J. Gore, H. Youk, and A. van Oudenaarden, ibid. 459, 253 (2009).

[5] D. Semmann, H. J. Krambeck, and M. Milinski, Nature (London) 425, 390 (2003); A. Traulsen, D. Semmann, R. D. Sommerfeld, H. J. Krambeck, and M. Milinski, Proc. Natl. Acad. Sci. USA 107, 2962 (2010).

[6] M. Doebeli and C. Hauert, Ecol. Lett. 8, 748 (2005); M. A. Nowak, Science 314, 1560 (2006).

[7] J. A. Fletcher and M. Zwick, J. Theor. Biol. 228, 303 (2004); W. D. Hamilton, ibid. 7, 1 (1964); 7, 17 (1964); D. S. Wilson, Proc. Natl. Acad. Sci. USA 72, 143 (1975); A. Traulsen and M. A. Nowak, ibid. 103, 10952 (2006).

[8] R. L. Trivers, Quart. Rev. Biol. 46, 35 (1971); M. A. Nowak and K. Sigmund, Nature (London) 364, 56 (1993); L. A. Imhof, D. Fudenberg, and M. A. Nowak, Proc. Natl. Acad. Sci. USA 102, 10797 (2005); M. A. Nowak and K. Sigmund, Nature (London) 437, 1291 (2005); L. A. Imhof, D. Fudenberg, and M. A. Nowak, J. Theor. Biol. 247, 574 (2007); J. M. Pacheco, A. Traulsen, H. Ohtsuki, and M. A. Nowak, ibid. 250, 723 (2008); A. J. Bladon, T. Galla, and A. J. McKane, Phys. Rev. E 81, 066122 (2010).

[9] M. A. Nowak and K. Sigmund, Nature (London) 393, 573 (1998); R. Ferrière, ibid. 393, 517 (1998); O. Leimar and P. Hammerstein, Proc. R. Soc. London B 268, 745 (2001); K. Panchanathan and R. Boyd, J. Theor. Biol. 224, 115 (2003); H. Ohtsuki and Y. Iwasa, ibid. 231, 107 (2004).

[10] M. A. Nowak and R. M. May, Nature (London) 359, 826 (1992); C. Hauert and G. Szabó, Complexity 8, 31 (2003); C. Hauert and M. Doebeli, Nature (London) 428, 643 (2004); F. C. Santos and J. M. Pacheco, Phys. Rev. Lett. 95, 098104 (2005); H. Ohtsuki and M. A. Nowak, J. Theor. Biol. 243, 86 (2006); 251, 698 (2008); C. E. Tarnita, H. Ohtsuki, T. Antal, F. Fu, and M. A. Nowak, ibid. 259, 570 (2009); Z. Wang, A. Szolnoki, and M. Perc, Sci. Rep. 2, 369 (2012); M. Assaf and M. Mobilia, arXiv:1202.3231v1.

[11] C. Hauert, S. De Monte, J. Hofbauer, and K. Sigmund, Science 296, 1129 (2002); J. Theor. Biol. 218, 187 (2002); C. Hauert, A. Traulsen, H. Brandt, M. A. Nowak, and K. Sigmund, Science 316, 1905 (2007); M. Perc and A. Szolnoki, New J. Phys. 14, 043013 (2012).
[12] J. F. Crow and M. Kimura, An Introduction to Population Genetics Theory (Blackburn Press, Caldwell, New Jersey, 2009); W. J. Ewens, Mathematical Population Genetics (Springer, New York, 2004).

[13] M. A. Nowak, A. Sasaki, C. Taylor, and D. Fudenberg, Nature (London) 428, 646 (2004); C. Taylor, D. Fudenberg, A. Sasaki, and M. A. Nowak, Bull. Math. Biol. 66, 1621 (2004).

[14] T. Antal and I. Scheuring, Bull. Math. Biol. 68, 1923 (2006).

[15] A. Traulsen, J. C. Claussen, and C. Hauert, Phys. Rev. Lett. 95, 238701 (2005); A. Traulsen, J. M. Pacheco, and L. A. Imhof, Phys. Rev. E 74, 021905 (2006); A. Traulsen, J. C. Claussen, and C. Hauert, ibid. 74, 011901 (2006); M. Mobilia, Europhys. Lett. 95, 50002 (2011).

[16] Such a formulation is commonly used since it represents the most convenient way to capture the essential features of the classical PD [10]. It is an example of "equal gains from switching" games; see, e.g., [2] and references therein.

[17] C. W. Gardiner, Handbook of Stochastic Methods (Springer, New York, 2002); H. Risken, The Fokker-Planck Equation (Springer, New York, 1989).

[18] N. G. van Kampen, Stochastic Processes in Physics and Chemistry (Elsevier, Amsterdam, 1997).

[19] L. E. Blume, Games Econ. Behav. 5, 387 (1993); G. Szabó and C. Töke, Phys. Rev. E 58, 69 (1998); C. Hauert and G. Szabó, Am. J. Phys. 73, 405 (2005); A. Traulsen, M. A. Nowak, and J. M. Pacheco, Phys. Rev. E 74, 011909 (2006).

[20] See, e.g., M. Mobilia and M. Assaf, Europhys. Lett. 91, 10002 (2010); M. Assaf and M. Mobilia, J. Stat. Mech. (2010) P09009; J. Theor. Biol. 275, 93 (2011), and references therein.

[21] P. A. P. Moran, The Statistical Processes of Evolutionary Theory (Clarendon, Oxford, 1962).

[22] When the density of facilitators is $z=r+(1-r) / N$, the selective pressure due to the cost-to-benefit ratio is balanced and one has $v_{N}=0$. In this special case, the dynamics is neutral (there is effectively no selection) with $T_{j}^{+} / T_{j}^{-}=1$, and Eq. (6) yields the expected result $\phi_{j, v_{N}=0}^{\mathrm{C}}=$ $j /(N-\ell)=x /(1-z)$.

[23] D. T. Gillespie, J. Comput. Phys. 22, 403 (1976). 